UCRL-TR-234930

LCLS-TN-07-11

September 28, 2007

\title{
Space-charge effects in a gas detector
}

\author{
D.D. Ryutov, S. Hau-Riege, R.M. Bionta \\ Lawrence Livermore National Laboratory, Livermore, CA 94550
}

\begin{abstract}
Discussion of space-charge effects in a photoluminescence cell that will be used as a nondisruptive total energy monitor at the LCLS facility is presented. Regimes where primary photoelectrons will be confined within the X-ray beam aperture are identified. Effects of the space-charge on the further evolution of the electron and ion populations are discussed. Parameters of the afterglow plasma are evaluated. Conditions under which the detector output will be proportional to the pulse energy are defined.
\end{abstract}

\section{Disclaimer}

This document was prepared as an account of work sponsored by an agency of the United States Government. Neither the United States Government nor the University of California nor any of their employees, makes any warranty, express or implied, or assumes any legal liability or responsibility for the accuracy, completeness, or usefulness of any information, apparatus, product, or process disclosed, or represents that its use would not infringe privately owned rights. Reference herein to any specific commercial product, process, or service by trade name, trademark, manufacturer, or otherwise, does not necessarily constitute or imply its endorsement, recommendation, or favoring by the United States Government or the University of California. The views and opinions of authors expressed herein do not necessarily state or reflect those of the United States Government or the University of California, and shall not be used for advertising or product endorsement purposes.

This work was performed under the auspices of the U.S. Departent of Energy by University of California Lawrence Livermore National Laboratory under contract No. W-7405-Eng-48. Work supported in part by the DOE contract DE-AC02-76SF00515. This work was performed in support of the LCLS project at SLAC. 


\section{Introduction}

In this note we consider space-charge effects in the photoluminescence cell [1] which will be used for non-destructive measurements of the total energy of X-ray pulses on the LCLS facility [2]. These effects have been briefly discussed in our earlier reports $[3,4]$. Here we provide a more detailed analysis.

We use the term "pulse energy" and symbol " $Q$ " to designate the total energy content of one X-ray pulse. To designate the energy of X-ray quanta, we use the symbol " $E_{X}$ " and the term "X-ray energy".

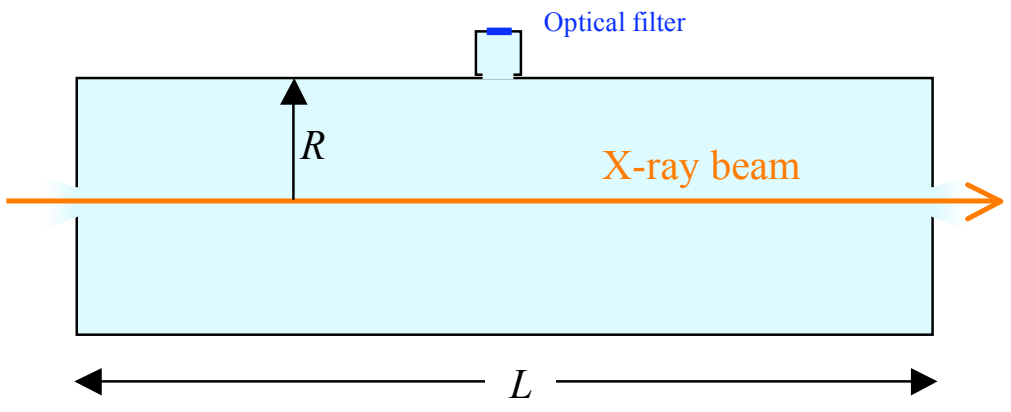

Fig.1 Schematic of the photoluminescence cell. The visible and UV light created in the cell passes through the optical filter and hits a detector (not shown). At a given fill pressure and X-ray energy $E_{X}$ the detector signal is the measure of the pulse energy $Q$.

A schematic of the photoluminescence cell is shown in Fig. 1. The cell is a cylinder filled with nitrogen gas of an adjustable pressure. At the end walls there are two orifices that open to the differential pumping system. The orifice radius is $a=0.15 \mathrm{~cm}$, the cell length is $L=30 \mathrm{~cm}$ [1]. The gas inlet is not shown. The X-ray beam enters and exits the cell through the orifices; on its way through the gas it creates, predominantly by the photoionization, the "primary" electrons which have initial kinetic energy comparable to the X-ray energy. These primary electrons then spend their energy to produce excitations and secondary ionizations; the secondary electrons are much more numerous but have a much lower energy $\sim$ a few electron-volt. Significant fraction of excited states decays radiatively, producing UV and optical radiation that can be detected, e.g., by a photomultiplier tube situated outside the cell. To prevent the primary electrons from hitting the side walls, a weak axial magnetic field will be imposed (see [1]).

We show that, for the lower end of the X-ray energies, between 0.8 and $2.5 \mathrm{keV}$, space charge may lead to confinement of the "primary" photoelectrons within the beam radius. The secondary electrons and excited atomic particles are then also generated mostly in this very narrow zone, irrespectively to the presence of the guiding magnetic field. This space-charge confinement of the primary electrons takes place even at the pulse energies well below the design value of $2 \mathrm{~mJ}$, down to $\sim 100 \mu \mathrm{J}$.

At higher X-ray energies, between 2.5 and $8 \mathrm{keV}$, the electrostatic confinement of the "primary" photoelectrons is weak, and they produce ionization and excitation in a much broader zone. However, space-charge effects play a substantial role in the dynamics of the secondary electrons. Unless the pulse energy is very small, below $1 \mu \mathrm{J}$, the density of the secondary electrons and neutralizing ions is such that they can be considered as a quasineutral plasma. This conclusion affects estimates of the particle diffusion to the walls and the recombination time. We find that, under typical conditions, the decay time of this plasma is longer than the time interval between two successive Xray pulses. Therefore, this plasma is continuously present in the cell, generating a steady- 
state low-intensity radiation, which, in principle, can be used for the independent evaluation of the average pulse energy. The overall time-dependence of the radiation from the detector is qualitatively shown in Fig. 2, where short bursts of radiation are followed by a low-intensity afterglow. It should be noted that the cool quasineutral plasma is formed also in the aforementioned case of electrostatic confinement of primary electrons.

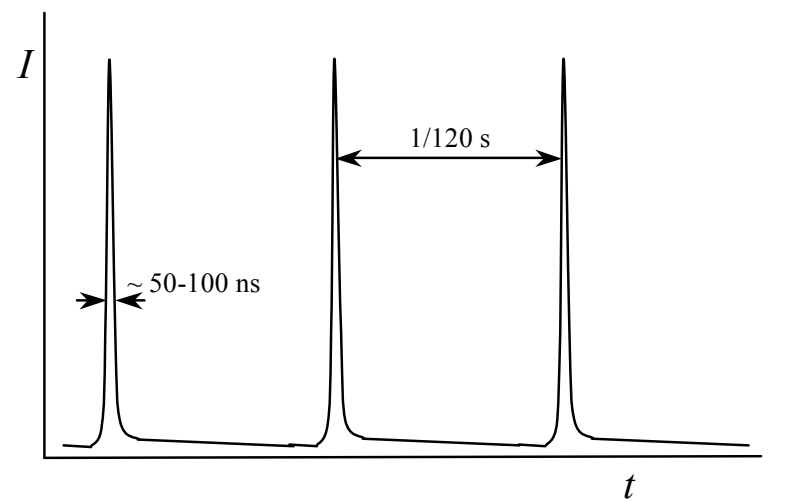

Fig. 2 A qualitative picture of the timedependence of the radiation intensity $I$, arbitrary units. There is a continuous background radiation with short spikes superimposed on it. The total energy radiated between two pulses (predominantly recombination radiation) can be comparable with the total energy radiated in each pulse.

In what follows, we are not attempting to produce "exact" results, but rather to provide a quick order-of-magnitude scoping study. In Sec. 2, we derive conditions under which electrostatic confinement of the primary electrons is present. In Sec. 3, we consider characteristic time-scales describing the primary electrons. In Sec. 4 we find the number of "secondary" charged particles produced by the impact ionization of the molecules by the primary electrons and show that space-charge effects are very strong at this stage. In Sec. 5, we evaluate the volume density of the secondary electrons and conclude that the ionization degree, by the time of arrival of the next X-ray pulse, is small, so that the interaction of every successive X-ray pulse with the gas is not affected by the perturbations created by the previous pulse. In Secs. 6-8 we consider the physics of the "afterglow" plasma that is present in the cell between the X-ray pulses. The role of this cold recombining plasma in generating quasi-steady, low intensity "afterglow" is discussed. Finally, in Sec. 9 we discuss our results in terms of possible modes of operation of the gas detector.

\section{Electrostatic confinement of the primary electrons}

Consider an electric potential created by a bare ion cylinder of a radius $r$ situated inside a conducting grounded cylinder of a radius $R$. This simple model allows us to get some insights into the possible significance of space-charge effects: if the resulting potential well (for the electrons) is much deeper than their characteristic energy, this would mean that the electrons will be bound to the ion core, and we will actually deal with a quasineutral plasma. The potential on the axis of a charged cylinder of a radius $r$ is

$$
\varphi=q\left(1+2 \ln \frac{R}{r}\right)
$$


where $q$ is the charge per unit length. For the ions formed initially within the radius of the X-ray beam, $r$ should be identified with the beam radius. The potential difference between the axis and the distance $\sim 2 r$ from the axis is

$$
\Delta \varphi=q(1+2 \ln 2) \approx 2.4 q
$$

The primary electrons will be confined within the radius $\sim 2 r$ if their energy is less than $e \Delta \varphi$.

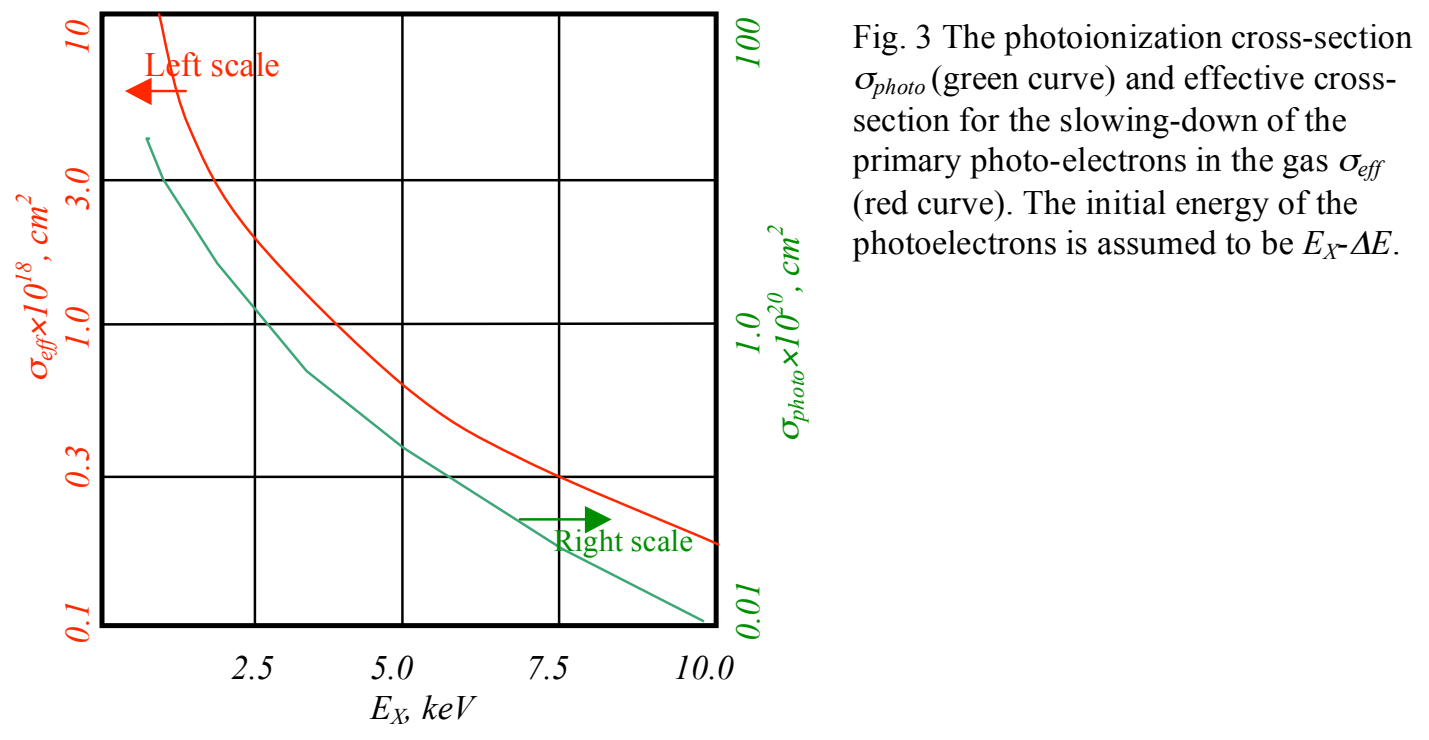

The number of the primary ions produced by the X-ray beam per unit length is:

$$
\tilde{N}_{i}^{(p)}=n_{0} \sigma_{p h} N_{X}
$$

where $n_{0}$ is the neutral gas particle density, $\sigma_{p h}$ is the photoionization cross-section for the incident X-ray beam, $N_{X}$ is the number of X-ray photons in one pulse, the superscript " $p$ " stands for the word "primary", and we use the "tilde" sign to designate the quantities related to a unit length, in particular, $\tilde{N}_{i}^{(p)}$. So, the potential well for the primary electrons will be roughly

$$
U=e \Delta \varphi \approx 2 e^{2} n_{0} \sigma_{p h} N_{x}
$$

(we rounded the numerical coefficient 2.4 in Eq. (2) to 2, to emphasize the qualitative nature of our analysis). For reference purposes, the plot of the photo-ionization crosssection (Ref. 5) is shown in Fig. 3 (right-hand scale).

The coefficient $n_{0} \sigma_{p h}$ is approximately equal to $\varepsilon / L$, where $\varepsilon$ is a fraction of the energy lost by the X-ray pulse in the cell, and $L$ is the length of the cell:

$$
\varepsilon \approx \sigma_{p h} n_{0} L
$$


Not to interfere with the downstream experiments with the X-ray beam, the allowed loss of X-ray photons in the detector is set at the level of $5 \%$, maximum [1]. For the $30-\mathrm{cm}$ long cell accepted in the current design [1], the condition $\varepsilon=0.05$ corresponds to the dashdotted line in Fig. 4. For $E_{X}$ exceeding, roughly, $2 \mathrm{keV}$, this curve lies above 2 torr, which pressure is adopted as the maximum allowable pressure in the detector (not to overburden the differential pumping system). The horizontal dashed line near the top of Fig. 4 corresponds to $p=2$ torr. So, the operational domain of the detector in the $\left(E_{X}, p\right)$ plane is determined by the condition $\varepsilon<0.05$ at $E_{X}<2 \mathrm{keV}$, and by the condition $p<2$ torr at $E_{X}>2$ $\mathrm{keV}[1]$.

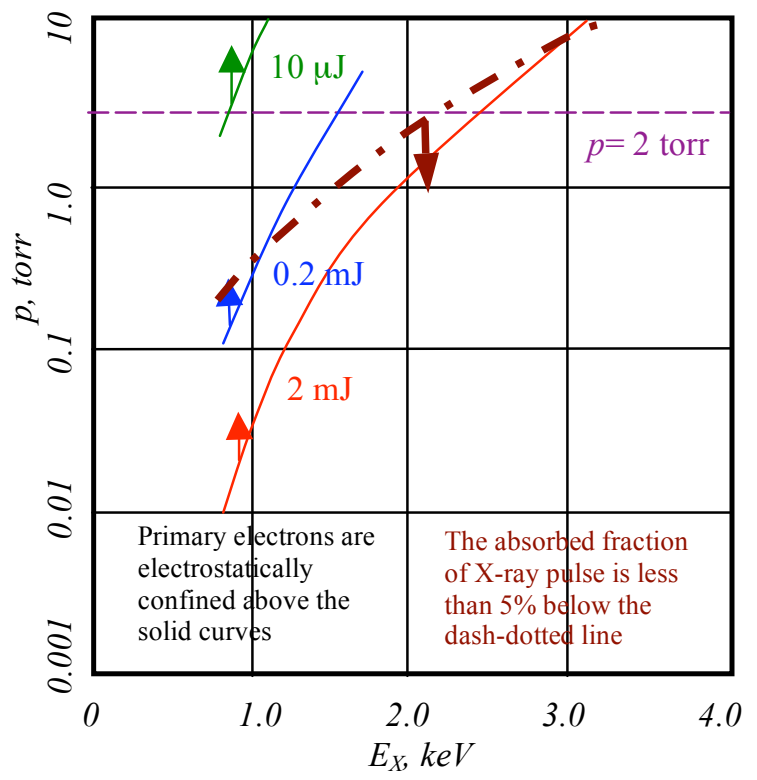

Fig. 4 On the role of the electrostatic confinement of the primary electrons. The dash-dotted line corresponds to the absorption of $5 \%$ of the initial X-ray pulse energy. Three solid lines correspond to the onset of electrostatic confinement for three pulse energies: $2 \mathrm{~mJ}$ (red curve), $0.2 \mathrm{~mJ}$ (blue curve), and $0.01 \mathrm{~mJ}$ (green curve).

The number of X-ray quanta per pulse is

$$
N_{X}=6.4 \times 10^{12} \frac{Q(\mathrm{~mJ})}{E_{X}(\mathrm{keV})} .
$$

The number density of the nitrogen molecules is related to the fill pressure $p$ :

$$
n_{0}\left(\mathrm{~cm}^{-3}\right)=3.5 \times 10^{16} \mathrm{p}(\text { torr }),
$$

The primary electrons have the energy that is smaller than the X-ray energy by some amount $\Delta E$ (on average); following Ref. 1, we assume that $\Delta E=0.4 \mathrm{keV}$, roughly corresponding to the K-shell energy. In other words, the initial energy of the primary electrons is $E_{X}-\Delta E$, and condition for the electrostatic confinement of the primary electrons can be represented as $U>E_{X}-\Delta E$. In "practical" units this condition reads as (see Eqs. (4), (6), (7)):

$$
p(\text { torr })>\frac{7.5 \times 10^{-21} E_{X}(\mathrm{keV})\left[E_{X}(\mathrm{keV})-0.4\right]}{\sigma_{p h}\left(\mathrm{~cm}^{2}\right)} .
$$


It is illustrated in Fig. 4. Note that confining potential $\Delta \varphi$ isolates the primary electrons not only from the side walls but also from the end walls (provided they have the same potential as the side wall). Figure 4 shows that, for the pulse energy of $2 \mathrm{~mJ}$ and the pressure determined by the $\varepsilon=0.05$ constraint, the electrostatic confinement of the primary electrons occurs for the X-ray energy below, roughly, $2.5 \mathrm{keV}$. For the 10-times smaller pulse energy, the confinement is present for $E_{X}<1 \mathrm{keV}$.

Within the time during which the primary electrons produce impact ionization and lose their energy (a few nanoseconds under typical conditions, see Sec. 3), the ions are not displaced by any significant distance. Therefore, the secondary electrons are also confined to the narrow channel.

\section{Characteristic spatial and temporal scales for the primary electrons}

The primary electrons, after having been born near the axis of the cell, lose their energy on the ionization and excitation. In the course of the energy loss, they also get scattered, so that their trajectory is not a straight line. This complex set of processes could be roughly characterized by the parameter $D_{\text {eff, }}$, the range, of the primary electrons, which is basically their 1D penetration depth into the half-space filled with the matter in question. For gases, the range is inversely proportional to the particle density $n_{0}$; it is then convenient to introduce the parameter of the dimension of cross-section $\sigma_{\text {eff }}$ related to the range as

$$
\sigma_{e f f}=\frac{1}{n_{0} D_{e f f}} .
$$

We introduce also the quantity $\tau_{\text {eff }}$ of the dimension of time, which is defined as $D_{\text {eff }} / \mathrm{v}_{0}$,

$$
\tau_{e f f}=\frac{D_{e f f}}{\mathrm{v}_{0}},
$$

where $\mathrm{v}_{0}$ is the initial velocity of the electron,

$$
\mathrm{v}_{0}=\sqrt{2\left(E_{X}-\Delta E\right) / m} .
$$

The plot of $\sigma_{\text {eff }}$ based on Ref. 6 is shown in Fig. 3 (left-hand scale). Note that $\sigma_{e f f}$ is roughly $50-100$ times higher than the photoabsorption cross-section.

With axial magnetic field imposed, primary electrons do not reach the side walls of the cylindrical cell. They may hit the end walls, if they are not electrostatically confined. Depending on the range $D_{\text {eff }}$ the smaller or larger fraction of the primary electrons leaves the volume before having lost their energy on excitation and ionization. When the range is large, the primary electrons are present in the middle section of the cell (where the optical detectors are situated), for the time $\sim L / 2 \mathrm{v}_{0}$, where the factor " 2 " accounts for the presence of two ends. [We consider here the situation where the detector field of view in the direction along the cell is much smaller than the cell length $L$.] As the primary photo-electrons are born with a relatively small parallel velocity, we suggest to 
use, as a characteristic axial loss velocity, not $\mathrm{v}_{0}$, but $\mathrm{v}_{0} / \sqrt{2}$. With that, we end up with the following estimate for the life-time with respect to un-inhibited parallel loss:

$$
\tau_{\|}=\frac{L}{\mathrm{v}_{0} \sqrt{2}}
$$

The life-time $\tau$ of the primary photoelectrons $\tau$ can then be evaluated by means of the equation

$$
\frac{1}{\tau}=\frac{1}{\tau_{\|}}+\frac{1}{\tau_{e f f}}
$$

that accounts for the both loss channels.

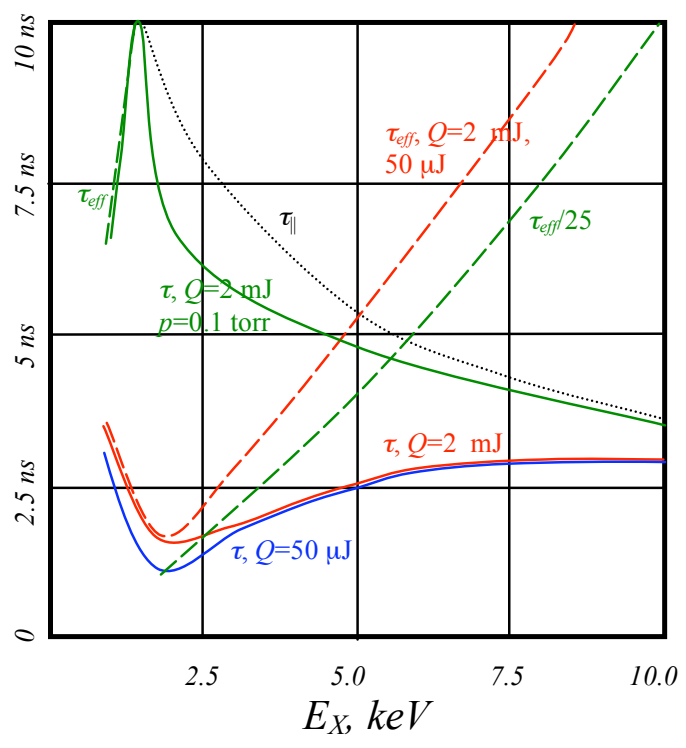

Fig. 5 Characteristic times for the primary electrons. The black dotted line is the time of the (uninhibited) parallel losses $\tau_{\|}$. Dashed and solid lines depict $\tau_{\text {eff }}\left(E_{X}\right)$ and $\tau\left(E_{X}\right)$, respectively. Red (blue) lines correspond to the pulse energy $Q=2 \mathrm{~mJ}$ $(Q=50 \mu \mathrm{J})$ and the pressure determined by condition $\varepsilon=0.05$ (Eq. (5)) for $E_{X}<2 \mathrm{keV}$ and $p=2$ torr for $E_{X}>2 \mathrm{keV}$. The curves for $\tau_{\text {eff }}$ for the cases of $Q=2 \mathrm{~mJ}$ and $Q=50 \mu \mathrm{J}$ are the same, and only one of them is shown. Green lines correspond to $Q=2 \mathrm{~mJ}$ and a low pressure of 0.1 torr over the whole range of X-ray energies. The low-energy peak on green solid line is caused by the effect of the electrostatic confinement. Note the change of scale for the right part of the dashed green line.

The plots of $\tau_{\|,}, \tau_{\text {eff }}$ and $\tau$ are presented in Fig. 5. By its definition, the parallel loss time (12) (black dotted line in Fig. 5) does not depend on the gas pressure. The red curves for $\tau_{\text {eff }}$ and $\tau$ correspond to the pressure that is determined by Eq. (5) for $E_{X}<2$ $\mathrm{keV}$, and $p=2$ torr for $E_{X}>2 \mathrm{keV}$. The pulse energy for these curves is assumed to be $2 \mathrm{~mJ}$. This determines the X-ray energy range for which the electrostatic confinement is present. As discussed in Sec. 2, the electrostatic confinement exists (for the assumptions regarding the gas pressure that we made) for $E_{X}<2.5 \mathrm{keV}$. In this energy range, the axial losses are absent, and $\tau_{\|}$in Eq. (13) should be replaced by infinity. This is why the lowerenergy branch of the $\tau\left(E_{X}\right)$ curve overlaps with the $\tau_{e f f}\left(E_{X}\right)$ curve. For reference purpose, we present also the same set of curves (blue lines) for a significantly smaller pulse energy, $Q=50 \mu \mathrm{J}$, where there is no electrostatic confinement at any energy. Also, for reference purpose, we present curves (green lines) for the case where the pulse energy is $2 \mathrm{~mJ}$, but the pressure is small, 0.1 torr, over the whole range of X-ray energies.

The primary electron population in the location of the detector decays with a characteristic time $\tau$. As this time is generally quite short, the spatial distribution of the 
ions does not change significantly during this time. In other words, the radial size of the ionized column by the time when the primary electrons already gets "communalized" with the much denser population of secondary electrons (which initially would have an average energy $\sim$ a few electron-volt), would be entirely determined by the spatial distribution of the ionizing (primary) electrons, on the background of the resting ions. Much later in time, the ion spatial distribution starts to evolve due to diffusion and recombination (see below).

\section{Space-charge effects for the secondary electrons}

As we have shown in the previous section, the primary electrons exist in the detector location within the characteristic time $\sim 10 \mathrm{~ns}$ for any realistic choice of the input parameters. Beyond this time, we deal with much colder electron population, with a characteristic energy $E^{(s)}$ of a few electron-volt (i.e., below the excitation threshold). The superscript " $s$ " here and below refers to the secondary particles. For these low-energy electrons space-charge effects are quite strong, so that they, together with the ions, form a quasineutral plasma.

The quasineutrality condition can be formulated based on Eq. (2) and reads as:

$$
2 e^{2} \tilde{N}_{i}^{(s)}>E^{(s)}
$$

where $\tilde{N}_{i}^{(s)}$ is the number of secondary ions produced per unit length of the cell. We use the "hat" symbol to designate the quantities related to a unit length. In "practical" units this condition becomes:

$$
\tilde{N}_{i}^{(s)}\left(\mathrm{cm}^{-1}\right)>3.5 \times 10^{6} \mathrm{E}^{(s)}(\mathrm{eV})
$$

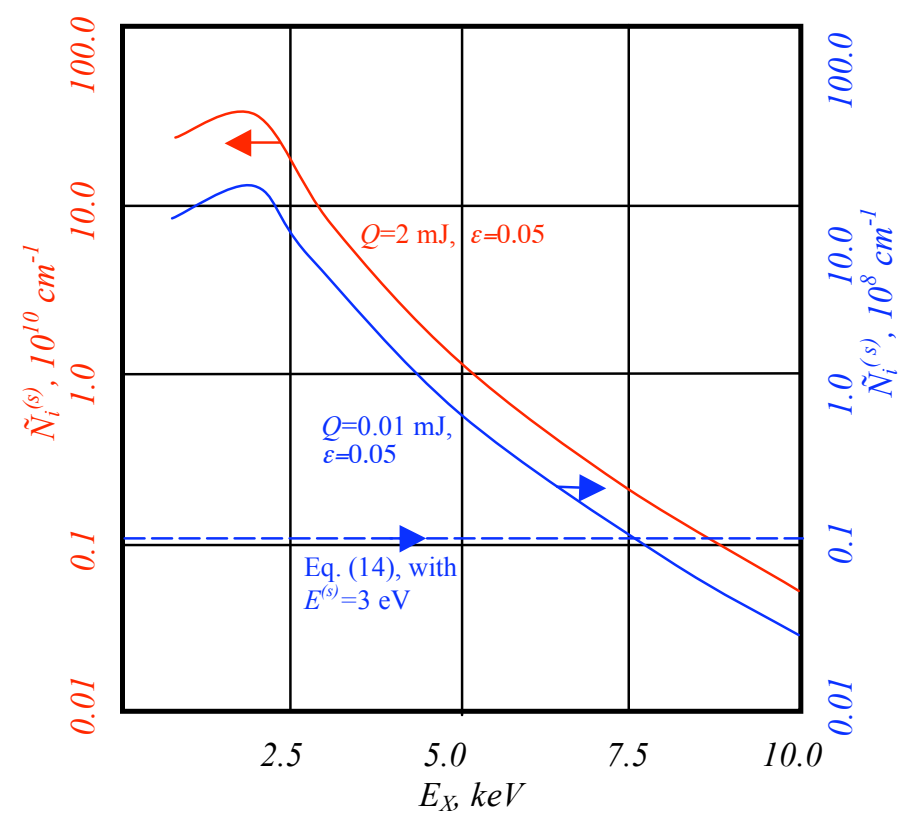

Fig. 6 The number of secondary ions per unit length of the cell, for $Q=2 \mathrm{~mJ}$ (red curve, left scale) and $Q=0.01 \mathrm{~mJ}$ (blue curve, right scale). In both cases, at $E_{X}<2 \mathrm{keV}$ a constraint of $\varepsilon=0.05$ is imposed; at $E_{X}>2 \mathrm{keV}$ the pressure is 2 torr. For the red curve, electrostatic confinement of primary electrons is important, whereas for the blue curve it is absent. Dashed line corresponds to the right scale and represents condition (14) for $E^{(s)}=3$ eV.

To estimate $\tilde{N}_{i}^{(s)}$ we note that the energy cost of producing one pair by the primary electron is $E_{\text {pair }} \sim 50 \mathrm{eV}$, i.e., higher than the ionization potential for nitrogen, 
because the primary electrons lose their energy not only on the ionization but also on the excitation of the neutral gas. The fraction of the primary electrons that lose their energy in the gas before hitting the walls is $\tau / \tau_{\text {eff }}=\tau_{\|} /\left(\tau_{\|}+\tau_{e f f}\right)$. Therefore, the initial number of secondary ions (and electrons) per unit length can be evaluated as

$$
\tilde{N}_{i}^{(s)} \sim \frac{n_{0} \sigma_{p h} \tau_{\|}}{\tau_{\|}+\tau_{e f f}} \times \frac{E_{X}-\Delta E}{E_{p a i r}} N_{X} .
$$

The plot of $\tilde{N}_{i}^{(s)}$ vs $E_{X}$ is shown in Fig. 6 for the following set of numerical parameters: $E_{\text {pair }}=50 \mathrm{eV}$, and $\Delta E=0.4 \mathrm{keV}$. In a broad range of input parameters the condition (2) is satisfied, i.e., the initial ionized state is that of a quasineutral plasma. The quasineutrality constraint is violated only at a very low pulse energy and high X-raqy energy, and/or at a very low gas pressure.

Excited states formed during the time $\tau$ (a few nanoseconds) decay within the time $\sim 50-100 \mathrm{~ns}$ [7] and produce UV and visible radiation, i.e., the useful signal. Within this radiation decay time no significant spatial redistribution of the excited molecules and atoms can take place. In other words, the spatial distribution of this 50-100 ns long radiation pulse mimics the initial energy deposition profile by the primary electrons. As we will see shortly, this 50-100 ns long pulse is followed by a long afterglow, which is caused by a slow recombination of the ionized component.

\section{The ion density}

In addition to the linear density of the primary and secondary ions, it is important to know also their volumetric densities, $n_{i}^{(p)}$ and $n_{i}^{(s)}$. To find the first, we have to divide $\tilde{N}_{i}^{(p)}$ (Eq. (3)) by the cross-section of the X-ray beam, $\pi a_{X}^{2}$ where $a_{X}$ is the beam radius, $n_{i}^{(p)}=\tilde{N}_{i}^{(p)} / \pi a_{X}^{2}$. With respect to the beam radius, we will use the following rough extrapolation:

$$
a_{X}(\mu m)=\frac{460}{\sqrt{E_{X}(k e V)}}
$$

which approximates reasonably well the beam radius at both low and high energies. Collecting Eqs. (3), (6), and (17), we find:

$$
\frac{n_{i}^{(p)}}{n_{0}}=1.1 \times 10^{15} \sigma_{p h}\left(\mathrm{~cm}^{2}\right) Q(\mathrm{~mJ})
$$

The significance of this ratio is related to the fact that the rear part of the X-ray pulse propagates already through the partially ionized gas, with the ionization degree determined by Eq. (18). The absorption coefficient for the rear part is somewhat different from that of the front part, which difference, if it was large enough, might affect our conclusions. However, as one sees from Eq. (18) and Fig. 3, even at the pulse energy of 2 $\mathrm{mJ}$ and the highest photoabsorption cross-section (corresponding to the X-ray energy of $0.826 \mathrm{keV}$ ), the ratio is in the range of $10^{-3}$ and, therefore, negligible. 
We now proceed to the evaluation of the density of the secondary plasma. Here one has to distinguish between the case of a strong electrostatic confinement of the primary electrons (the zone above the red curve in Fig. 4) and the case where there is no electrostatic confinement. In the first case, the ionized zone occupies the radius that is only by a factor of 2 larger than the X-ray beam radius, $a^{(s)}=2 a_{X}$. Using Eqs. (6), (16), and (17), we find that in the first case

$$
n_{i}^{(s)}\left(\mathrm{cm}^{-3}\right)=2.8 \times 10^{14} \frac{\varepsilon}{L(\mathrm{~cm})} \frac{E_{X}-\Delta E}{E_{\text {pair }}} Q(\mathrm{~mJ})
$$

where $\varepsilon$ is defined by Eq. (5). As was explained in Sec. 5, the regime of a strong electrostatic confinement corresponds to $\tau_{\|}=\infty$ in Eq. (16), and we have taken this limit when deriving Eq. (19). The plot of $n_{i}^{(s)}$ is shown in Fig. 7 by red line for $\varepsilon=0.05, Q=2$ $\mathrm{mJ}$, and for the energy domain $E_{X}<2.5 \mathrm{keV}$, where, according to Sec. 2, the electrostatic confinement is strong. As before, the parameter $E_{\text {pair }}$ is taken to be $0.05 \mathrm{keV}$. One can check that the ionization degree remains smaller than $1 \%$ in all cases.

If one wants to operate under conditions where there is no electrostatic confinement at $E_{X}<2.5 \mathrm{keV}$ and $Q=2 \mathrm{~mJ}$, one has to reduce the pressure by a factor of 23 below the red curve in Fig. 4. One may want to do that to avoid non-linear effects in the optical/UV output (see Sec. 7).

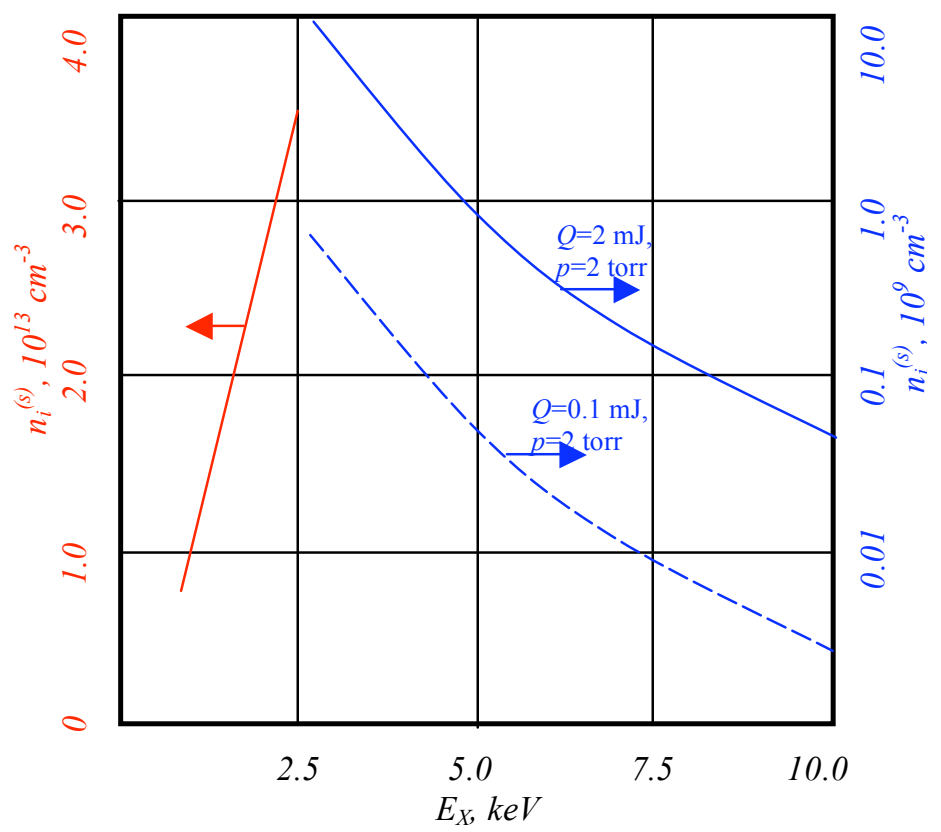

Fig. 7 The density of the secondary plasma immediately after the end of the ionization process (i.e., after $\sim 10$ ns). The red line (left scale) corresponds to the regime of electrostatic confinement and $\varepsilon=0.05$. The blue lines (right scale) correspond to the regimes with no electrostatic confinement. Note that the left scale is linear, whereas the right one is logarithmic.

In the regimes with no electrostatic confinement of the primary electrons, the radius of the ionized column is determined by the gyro-radius of the primary electrons. As a representative value, we take simply $a^{(s)}=2 \mathrm{~cm}$. In this regime, one obtains from Eq. (16) that 


$$
n_{i}^{(s)} \approx \frac{n_{0} \sigma_{p h} \tau_{\|}}{\tau_{\|}+\tau_{e f f}} \times \frac{E_{X}-\Delta E}{E_{p a i r}} \frac{N_{X}}{\pi a^{(s) 2}}
$$

Using Eqs. (6) and (7), and substituting $a^{(s)}=2 \mathrm{~cm}, E_{\text {pair }}=0.05 \mathrm{keV}$, one can transform this equation to

$$
n_{i}^{(s)}\left(\mathrm{cm}^{-3}\right)=3.57 \times 10^{9} \frac{\sigma_{p h}\left(10^{-20} \mathrm{~cm}^{2}\right) \tau_{\|}}{\tau_{\|}+\tau_{\text {eff }}} \times \frac{E_{X}(\mathrm{keV})-0.4}{E_{X}(\mathrm{keV})} Q(\mathrm{~mJ}) p(\text { torr })
$$

This result is illustrated in Fig. 7, for the domain $E_{X}>2.5 \mathrm{keV}$, by blue curves.

The ion component of this plasma will consist of molecular ions, $\mathrm{N}_{2}{ }^{+}$, and atomic ions $\mathrm{N}^{+}$; we neglect a small admixture of $\mathrm{N}_{2}{ }^{++}$. The ratio of the densities of $\mathrm{N}_{2}{ }^{+}$and $\mathrm{N}^{+}$is approximately $3[8,9]$. The composition may be important in the evaluation of the recombination rate. The ion temperature stays close to the gas temperature during all the times of interest for us.

This cold plasma is formed, as we have already mentioned, within 5-10 ns after the X-ray pulse. Its decay is determined by two processes: volumetric recombination and diffusion to the walls (where the surface recombination occurs). Some fraction of these recombinations gives rise to formation of excited molecular and atomic states, which then decay and produce radiation in the same wavelength range as the radiation emitted during the first burst. As we shall see, both recombination and diffusion processes take typically much longer time than $100 \mathrm{~ns}$ (except for the highest ion density case shown in Fig. 7), and produce a long afterglow after each X-ray pulse. In most cases, the time-scale for the decay of this afterglow is comparable to the pulse-to-pulse interval $(1 / 120 \mathrm{~s})$, so that a continuous glow is generated.

\section{Electron energy equilibration with the neutral gas}

The collisions with the gas molecules will gradually cool the electrons down. This takes time of roughly

$$
\tau_{\text {cooling }}=\frac{M}{m} \frac{1}{n_{0} \sigma_{e 0} \mathrm{v}_{T e}},
$$

where $m$ and $M$ are the electron and the molecule mass, respectively, and $\sigma_{\mathrm{e} 0}$ is the electron-molecule elastic cross-section. Taking as a representative value for the latter $10^{-15} \mathrm{~cm}^{2}$, we find that

$$
\tau_{\text {cool }}(s) \sim \frac{2.4 \times 10^{-5}}{p(\text { torr }) \sqrt{T_{e}(e V)}}
$$

For $p \sim 1$ torr, the cooling from $T_{e} \sim 3 \mathrm{eV}$ to $T_{e} \sim 1 \mathrm{eV}$ takes $\sim 10^{-5} \mathrm{~s}$, whereas cooling down from $1 / 20 \mathrm{eV}$ to $1 / 40 \mathrm{eV}$ (room temperature) takes $\sim 10^{-4} \mathrm{~s}$, i.e., the cooling down occurs well within the inter-pulse time interval. 


\section{Volumetric recombination}

For the molecular ions, the recombination process will be dominated by the dissociative recombination. According to Refs. [10,11, 12], this time can be roughly evaluated as

$$
\tau_{2}(s)=\frac{3 \times 10^{6}}{n_{i}^{(s)}\left(\mathrm{cm}^{-3}\right)} .
$$

This time can become comparable to the radiative decay time at the highest ion densities - those corresponding to the case of electrostatic confinement. Indeed for the ion density of $3 \times 10^{13} \mathrm{~cm}^{-3}$ (see Sec. 6), the dissociation recombination time becomes of order of 100 ns. Dissociative recombination may be accompanied by formation of excited atomic states, whose radiative decay would contribute to the UV-optical radiation signal. As our capability to make quantitative predictions of the processes involved is very limited, one may think that the regimes of a strong electrostatic confinement should be avoided, by reducing the gas pressure (see Sec. 2).

If one considers regimes where there is no electrostatic confinement, the recombination time becomes much longer. For example, for the density of $10^{8} \mathrm{~cm}^{-3}$, this time is $\sim 0.03 \mathrm{~s}$ and exceeds pulse-to-pulse interval.

For the atomic ions there is, obviously, no dissociative recombination. The radiative recombination is slow [13]:

$$
\tau_{r}(s)=\frac{3.7 \times 10^{12} \sqrt{T_{e}(e V)}}{n_{e}\left(\mathrm{~cm}^{-3}\right)}
$$

The 3-body recombination time at room temperature, $(1 / 40) \mathrm{eV}$, with the $3^{\text {rd }}$ body being another electron, is [13]:

$$
\tau_{3}(s)=\frac{7 \times 10^{18}}{\left[n_{i}\left(\mathrm{~cm}^{-3}\right)\right]^{2}} .
$$

For the ion density of $10^{8} \mathrm{~cm}^{-3}$, this time is orders of magnitude longer then the distance between the pulses.

The faster way for atomic ions to recombine may be multi-stage processes. In particular, one can expect a relatively high rate of the 3-particle process of the type $\mathrm{N}_{2}+\mathrm{N}^{+}+\mathrm{N}_{2} \rightarrow \mathrm{N}_{3}{ }^{+}+\mathrm{N}_{2}$, (see Eq. (172) in Ref. 14) with the subsequent dissociative recombination of the molecular ion. However, the kinetics of the processes involved is not known in sufficient detail and it is difficult at this point to make any reliable estimates. There are dozens of relevant processes with participation of ions, atoms, and molecules that may affect the state of the secondary plasma. Additional complexities to the "chemistry" of the secondary plasma may come from the formation of the negative nitrogen ions $\mathrm{N}_{2}{ }^{-}$. Developing a complete picture of the processes occurring in the afterglow plasma would require significant efforts. 


\section{Ambipolar diffusion}

In the cases where volumetric recombination is slow, the ionized state will decay by the diffusion of the ions to the walls and the surface recombination. This diffusion will occur at a rate determined by the ion mean-free path $\lambda_{i}$ with respect to elastic scattering on the neutrals and charge exchange process. As a representative value of this mean free path we take the following estimate:

$$
\lambda_{i}(\mathrm{~cm}) \sim \frac{10^{-2}}{p(\text { torr })}
$$

The diffusion coefficient at room temperature will then be (for nitrogen)

$$
D\left(\mathrm{~cm}^{2} / \mathrm{s}\right) \sim \frac{200}{p(\text { torr })}
$$

For a cylinder of a radius $R$, the diffusion time to the walls can be evaluated as

$$
\tau_{d i f f} \sim \frac{R^{2}}{6 D}
$$

or, in "practical" units,

$$
\tau_{\text {diff }}(s) \sim 10^{-3}[R(\mathrm{~cm})]^{2} p(\text { torr })
$$

For the expected range of pressures in the gas cell, the diffusion time is typically comparable with the distance between the successive pulses at $120 \mathrm{~Hz}$ rep rate. This means that there will be a continuous presence of the ionized component in the cell (unless the recombination is fast enough). The time of the gas replacement in the cell due to the continuous outflow through the $3-\mathrm{mm}$ diameter end apertures for the assumed radius of the cell $R=3 \mathrm{~cm}$ and $L=30 \mathrm{~cm}$ is of the order of $\tau_{\text {flow }} \sim 0.2 \mathrm{~s}$, much longer than $1 / 120$ s separation between the pulses.

In the presence of the axial magnetic field, the gyroradius $\rho_{e}$ of the secondary electrons will be small: after they cool down to the room temperature, it will be $\sim 30 \mu \mathrm{m}$ for the magnetic field of $200 \mathrm{G}$. This is much less than the electron mean free path at the gas densities of interest in our problem. The electron cross-field diffusion coefficient $D_{e}$ will then be of order of $\rho_{e}^{2} \mathrm{v}_{T e} / \lambda_{e}$ and may be significantly less than the ion diffusion coefficient (23), especially at lower gas densities. Then, due to the quasineutrality constraint, the radial diffusion may slow down. Regimes where the quasineutrality will be sustained due to parallel electron diffusion may become possible. We leave a more detailed analysis of these processes for the future work.

The interplay between the volumetric recombination and radial diffusion is important for the following reason. The charged particles that reach the wall, experience the surface recombination which, generally speaking, leads to formation of excited states

of neutral particles; radiative decay of these states then leads to an additional contribution 
to the quasi-steady-state radiation flux that would now come from the vicinity of the walls. This radiation source has obviously a different (from volumetric recombination) spatial distribution and may have also different spectrum. So, the correct interpretation of the afterglow requires the knowledge of the relative contribution of these two radiation sources. The previous sections provide initial material needed for such an assessment.

In the situation where the time for the cell to be cleared of the ionization is much longer than the time between the x-ray pulses, the density of the afterglow plasma will be determined by the average (over many pulses) source term $v s$ the decay term. In principle, this afterglow can be used to measure the average (over many pulses) energy of the pulses.

Of some concern may be chemical reactions with the wall material in the course of the surface recombination. This recombination may lead to formation of various chemical compounds made of the wall material and nitrogen. In the worst-case scenario, every ion impinging the wall would produce one molecule of such a compound (we will call these molecules "impurity molecules"). The number of ions produced per unit time per unit length is $\tilde{N}_{i}^{(s)} f$, where $f=120 \mathrm{~s}^{-1}$ is the repetition rate of X-ray pulses. Therefore, in the worst-case scenario, the number density of the molecules constantly present in the volume will be $\tilde{N}_{i}^{(s)} f \tau_{\text {flow }} / \pi R^{2}$. Taking $\tilde{N}_{i}^{(s)} \sim 10^{11}$, one finds that the volume density of impurities will be $\sim 10^{11} \mathrm{~cm}^{-3}$. This is significantly less than the density of the nitrogen molecules and would probably have no effect on the radiation processes.

Of some concern may be slow (within months of operation) erosion of the detector walls. Another concern is the presence of impurities in the exhaust system. Still, their amount is quite small and, in addition, one can control their composition by selecting the wall material.

\section{Discussion.}

At high pulse energy, $Q \sim 2 \mathrm{~mJ}$, and low X-ray energy, $E_{X}<2.5 \mathrm{keV}$, space-charge effects can confine primary electrons within the beam aperture. Then, the excited states are also formed in this narrow paraxial zone, thereby creating a very well defined radiation geometry. Another advantage of electron confinement is related to reduced electron end-loss (as the potential well is formed in the axial direction, too), Both these factors lead to increased detector signal. In extreme case of very high plasma density in the ionized channel, additional contribution to the useful signal may come from radiative decay of the excited states formed via recombination process.

One can extend this confinement regime towards higher X-ray energies by operating the cell at fill pressures higher than 2 torr, provided the differential pumping system could operate at these higher pressures.

A disadvantage of this mode is its possible non-linearity in the regimes where electrostatic confinement is marginal, in particular, at the higher X-ray energies approaching $2.5 \mathrm{keV}$. Here, the change of the pulse energy from, say, $2 \mathrm{~mJ}$ to $0.7 \mathrm{~mJ}$, would lead to the loss of confinement of the primary electrons, large expansion of the radiation zone, and change of the optical collection efficiency. At highest plasma density, the non-linearity may also come from the contribution of the recombination process.

Still, in the domain of $E_{X}<1.5 \mathrm{keV}$, the confinement is quite robust, and the 3-fold change of the pulse energy will not affect it. Also, in this regime, the density of the 
secondary plasma is below $1.5 \times 10^{13} \mathrm{~cm}^{-3}$, so that recombination during the optical pulse is less significant.

In the regimes where the electrostatic confinement of the primary electrons is absent, the visible/UV output during the first $100 \mathrm{~ns}$ burst is a linear function of the pulse energy $Q$, at given values of X-ray energy $E_{X}$ and fill pressure $p$. This conclusion is based on two observations: 1) In the absence of the electrostatic confinement of the primary electrons, the spatial distribution of excitation does not depend on the pulse energy; 2) The density of both primary and secondary plasmas in this regime is low enough not to create a nonlinear contribution to the radiation or to interfere with the processes occurring during subsequent pulses. The linearity of the output vs $Q$ could be of a significant help in the calibration process of the photoluminescence cell.

The afterglow signal, integrated over the distance between the pulses may be as large as the main pulse. It may contain contribution of the recombination radiation from the volume of the cell and from the walls. The ratio of the two is determined by the relative role of the ambipolar diffusion and volumetric recombination, as well as the chemical composition of the walls.

In this report, we have not discussed the role of various unwanted contributions to the detected signal, in particular, the contribution of the scattered X-ray (the scattering occurs with a cross-section of the order of Thomson cross-section). This process is relatively inefficient at the lower energies of $\mathrm{X}$ rays $(0.8-2.5 \mathrm{keV})$ but may become significant at the higher energies $\sim 8 \mathrm{keV}$. In principle, at these higher energies it can be used as a main process for evaluating the pulse energy. 


\section{References.}

1. S. Hau-Riege, R. Bionta, P. Duffy, K. Kishiyama, P. Pax, M. Roeben, D. Ryutov, S. Shen. "LCLS XTOD Gas Detector." DOE Review of the LCLS Project, October 25, 2006; S. Hau-Riege, P. Pax. "Gas Detector LCLS Engineering Specifications Document." LLNL Report UCRL-TR-228121, February 2007.

2. The LCLS Design Study Group. "Linac Coherent Light Source (LCLS) Design Study Report," Report SLAC-R-521 (Stanford University, Stanford, CA 1998).

3. D. Ryutov, R. Bionta, and S. Hau-Riege. "Photoluminescence for non-destructive total energy measurements and imaging of X-ray beam in the Linac Coherent Light Source," UCRL-TR-220017-DRAFT, March 2006.

4. D.D. Ryutov. "Some physics processes in the nitrogen-filled photoluminescence cell Rev. 1." UCRL-TR-222388 June 22, 2006

5. B.L. Henke, E.M. Gullikson, and J.C. Davis. "X-ray interactions: photoabsorption, scattering, transmission, and reflection at $\mathrm{E}=50-30000 \mathrm{eV}, \mathrm{Z}=1-92$." Atomic Data and Nuclear Data Tables, 54 (no.2), 181, 1993.

6. J.L. Barrett and P.B. Hays. "Spatial distribution of energy deposited in nitrogen by electrons." J. Chem. Phys., 64, 743 (1976).

7. A. Lofthus, P.. H. Krupenie "The spectrum of molecular nitrogen." Journal of Physical and Chemical Reference Data 6, Issue 1, pp. 113 (1977)

8. E. Krishnakumar and S.K. Srivastava. "Cross sections for the production of $\mathrm{N}_{2}^{+}$, $\mathrm{N}^{+}+\mathrm{N}_{2}{ }^{++}$and $\mathrm{N}^{++}$by electron impact on $\mathrm{N}_{2}$ ", J.Phys. B: At. Mol. Opt. Phys., 23, 1893 (1990).

9. H.C. Straub, P. Renault, B.G. Lindsay, K.A. Smith, R.F. Stebbings. "Absolute partial cross sections for electron-impact ionization of $\mathrm{H}_{2}, \mathrm{~N}_{2}$, and $\mathrm{O}_{2}$ from threshold to $1000 \mathrm{eV}$," Phys. Rev. A, 54, 2146 (1996).

10. H. Massey. "Atomic and molecular collisions." Taylor and Francis Ltd, London, 1979, p. 123.

11. J.B. Hasted. "Physics of atomic collisions," Butterworths, London, 1972, p. 423.

12. C.H. Sheehan, J.-P. St.-Maurice. "Dissociative recombination of $\mathrm{N}_{2}^{+}, \mathrm{O}_{2}{ }^{+}$and $\mathrm{NO}^{+}$: Rate coefficients for ground state and vibrationally excited ions." J. Geophys. Res., 109, A03302, 2004.

13. D.L. Book, NRL Plasma Formulary, NRL Publication 0084-4040, 1987.

14. I.A. Kossyi, A. Yu. Kostinsky, A.A. Matveyev, V.P. Silakov. Kinetic scheme of the non-equilibrium discharge in nitrogen-oxygen mixtures. Plasma Sources Sci. Technol. 1, 207 (1992). 